network to the patient and their carer and were able to bridge the gap between healthcare services and families. Carers of those with neurological conditions benefitted from having a dedicated person who understands the changing needs of their loved one and can provide them with some respite from caring responsibilities. Recommendations from the final report, available from August 2018, will be included in the poster.

\section{P-174 THE DEVELOPMENT OF AN MND CO-ORDINATOR ROLE AN EVALUATION OF THE FIRST YEAR}

Amanda Whitman, Lisa Pyrgoudes. Farleigh Hospice, Chelmsford, UK

\subsection{6/bmjspcare-2018-hospiceabs. 199}

The development of an MND co-ordinator role, an evaluation of the first year, the impact of the role for patients, carers and the extended multidisciplinary team.

Motor Neurone Disease (MND) is a rare neurological condition that is always fatal. It is a devastating and cruel illness, which affects seven in 100,000 people, for which there is no effective treatment. The quality of life of people living with MND can be dramatically improved through an effective model of co-ordinated care.

Farleigh is one of a handful of hospices in the UK to have a dedicated MND professional. This unique role was created to coordinate care and provide support within the community for those affected by MND within Mid Essex.

- The post-holder started October 2017, introducing patients early to the palliative MDT

- Providing a single point of contact within the community from diagnosis

- Working with patients, carers and health providers ensuring the needs for equipment, adaptations, therapy, information and support are met in a timely, cost efficient and effective way

- Patients and their families supported whilst navigating a vast array of health and social care services

- Peer support gym group for patients and carers

- Partnership working within local Hospital Trust, Regional MND Care and Research Centre, community based health and social care professionals and charitable organisations including the MND Association.

In 2016/17 there were 330 bed days attributed to those with MND in the local Acute Trust (approximately 25 patients). If we assume that the bed day cost on average is $£ 400$, then the total cost for the number of bed days occupied by MND patients in 2016/17 in Mid Essex was around $£ 132,000$. A key function of the role is to reduce reliance on secondary care input helping patients feel confident to remain in their own homes where appropriate.

\section{P-175 THE NEUROLOGICAL HUB}

Katie Margetts. Isabel Hospice, Welwyn Garden City, UK

10.1136/bmjspcare-2018-hospiceabs.200

Background Living with a neurological condition greatly impacts on quality of life (Cella, Nowinski, Peterman et al., 2011). 70\% of respondents in the 2016 Neurology Patient Experience Survey identify that living with a neurological condition frequently restricted engagement in activities ('Falling short: how has neurology patient experience changed since 2014?',Neurological Alliance, 2017; 'Parity of esteem for people affected by neurological conditions...', Neurological Alliance, 2017). The Neurological Alliance ('Parity of esteem for people affected by neurological conditions..., 2017) identifies significant gaps in neuropsychological and neuropsychiatric services.

Aims To develop a patient-centred, supportive 'hub' for those living with complex neurological conditions. To meet physical, mental, social, psychological and spiritual needs to enhance quality of life to live and die well.

Method Increasing demands for neurological palliative rehabilitation presented an opportunity to develop neurological services. A two-hour once a week group was piloted to develop a central 'hub' for patients and their families to access holistic support throughout the patient journey. Funded by charity.

The programme includes components that endorse self-management strategies, increase function and support wellbeing (Tiberini \& Richardson, 2015). Rehabilitation for those with palliative neurological conditions focuses on a multi-disciplinary holistic approach to enhance quality of life for patients and their families (Tiberini \& Richardson, 2015; National Institute for Health and Care Excellence, 2016). Therefore, group work and one-to-one therapies including mindfulness, exercise, art therapies, complementary therapies and social support are offered. Advance care planning and referrals to external supportive services are addressed.

Standardised quantitative outcome measures from The Outcome Assessment and Complexity Collaborative (Witt, Murtagh, de Wolf-Linder et al, 2016) are implemented. Qualitative word clouds are also collated to illustrate benefits to patients and their families.

Results The hub has been established for ten months with an average of 17 patients plus a family member attending weekly. Word clouds identify that patients and families feel; relaxed, positive, valued, supported and inspired. In addition to finding the hub friendly, social, helpful and calm.

Conclusion The neurological hub offers unique coordinated services to the patient life journey and beyond. The intention is to create a yearly programme offering increased opportunities and knowledge to patients and families.

\section{P-176 IMPROVING CARE COORDINATION FOR PEOPLE LIVING WITH MOTOR NEURONE DISEASE (MND) IN THE BRADFORD AREA}

${ }^{1}$ Kim Bonnar, ${ }^{2}$ Nicola Denbow, ${ }^{2}$ Sarah Holmes, ${ }^{2}$ Clare Rayment, ${ }^{2}$ Jenny White. ${ }^{1}$ Marie Curie, London, UK; ${ }^{2}$ Marie Curie, Bradford, UK

10.1136/bmjspcare-2018-hospiceabs.201

Background People living with Motor Neurone Disease (MND) frequently have care delivered by a high number of professionals. In the Bradford area, it was recognised that people living with MND did not have a single point of access for care. This was felt to lead to lower quality care through poor coordination and inequity in access to services.

Aim An MND Care Coordinator was appointed at a local hospice, with the aim of establishing a single point of access for patients and families. Additionally, the role aims to increase understanding of MND amongst professionals locally and support the running of the hospice multidisciplinary team clinics. 